\title{
Preparation and control of aligned cyclic rotational states
}

\author{
Juan Ortigoso, Delia Fernández-Torre, and Julio Santos \\ Instituto de Estructura de la Materia, CSIC, Serrano 121, 28006 Madrid, Spain
}

(Received 23 July 2013; published 20 September 2013)

\begin{abstract}
Molecules in selected rotational states can remain well aligned for extended periods of time in the presence of an appropriate periodic train of nonresonant laser pulses. Here, we show that these states can be prepared by slowly switching the electromagnetic field amplitude during a sequence of laser pulses. For low-temperature ensembles, a high degree of alignment can be achieved by designing pulse trains that take into account the distribution of avoided crossings between quasienergy curves. Additionally, we present calculations that illustrate several effects causing misalignment. A discussion of subtleties concerning systems with unbounded rotational spectra is also included.
\end{abstract}

DOI: 10.1103/PhysRevA.88.033418

PACS number(s): $33.80 . \mathrm{Wz}, 33.15 . \mathrm{Kr}, 32.80 . \mathrm{Qk}$

\section{INTRODUCTION}

The design of schemes to align molecular ensembles has become an active subfield of quantum control [1]. Appropriate external fields can excite molecules to rotational states that execute small-amplitude librations about the field direction. Friedrich and Herschbach [2] showed that these directional states correspond to instantaneous eigenstates of a molecule interacting with the envelope of a strong nonresonant laser pulse via its anisotropic polarizability. Within this model, the interaction between electrons and the laser field is represented by the molecular polarizability.

If the time evolution during the laser pulse is adiabatic, a molecule, initially in a field-free rotational eigenstate, is described at each time instant, during the pulse, by the corresponding instantaneous eigenstate. Thus, at the end of the pulse the molecule returns to the initial field-free eigenstate [3]. Depending on the duration of the laser pulse the dynamics can be nonadiabatic, and molecules end up in field-free aligned states. The components of these states subsequently dephase, and the alignment is quickly lost [3]. Alignment recurrences can take place under the further field-free evolution.

An alternative strategy allows us to maintain strong alignment over a substantial time [4]. These methods require us to use, instead of a single laser pulse, a periodic train of pulses. In this case, aligned states correspond to eigenstates of the Floquet operator, which is the time evolution operator during one period of the external field. It is particularly relevant that Floquet eigenstates are cyclic states. This means that a molecule which initially is in one Floquet eigenstate will return at the end of each pulse to the same state apart from an irrelevant phase factor. Thus, if a given Floquet eigenstate is well aligned during a single pulse, it remains aligned during a sequence of identical pulses.

The pertinent problem that we investigate here is how to create the initial Floquet eigenstates to be submitted to the periodic pulse train. No optimal strategy for this purpose has been demonstrated yet, although some methods have been suggested. Tailored microwave pulses could create cyclic states as shown before for other types of rotational wave packets [5]. However, a different microwave field for each initial energy eigenstate is needed. Another alternative is given by the switched-wave-packet method [6]. By suddenly switching off a long adiabatic pulse at a particular time, pendular wave packets can be obtained [7]. However, these states resemble cyclic states only for very short pulses.

Conceptual problems arise due to the fact that Floquet spectra are dense, and it is not clear beforehand if individual Floquet eigenstates can be prepared. We show that an efficient strategy for creating cyclic rotational states from field-free rotational states requires the design of an external field formed by laser pulses whose amplitude increases slowly from zero to a target amplitude. The variation of the laser intensity from pulse to pulse can be tailored in such a way that molecules are described during the whole sequence of pulses by a succession of single Floquet eigenstates. For appropriate laser parameters, a low-temperature field-free ensemble can be converted into an aligned ensemble.

Pulse trains that change slowly with time have been used before to execute population transfer in atoms [8]. Also, protocols for steering atomic Rydberg states by half-cycle pulses [9] have been designed. These methods employ a chirping frequency or a varying time delay between pulses of the train. Chirping schemes can be applied to our problem, but here, we only consider trains for which the field amplitude changes.

Slow switching of Floquet Hamiltonians is discussed in Sec. II. Results describing the preparation of cyclic aligned states and the high degree of control that can be achieved with our method are presented in Sec. III. Several effects that cause misalignment are discussed in Sec. IV. General conclusions are given in Sec. V. In Appendix A, basic notions concerning cyclic states are given. Appendix B presents a discussion of several issues that arise when the energy spectrum is unbounded.

\section{SLOW SWITCHING OF AN EXTERNAL PERTURBATION: ADIABATIC VERSUS DIABATIC SWITCHING}

The time evolution of a system $\mathcal{S}$ perturbed by an external field $\mathcal{E}$ that changes slowly with time can be fully adiabatic, fully diabatic, or intermediate, depending on the way that avoided crossings between the instantaneous energy levels of $\mathcal{S}+\mathcal{E}$ are traversed. In the first two cases, the system is described at all times by a single eigenstate of the Hamiltonian. In practice, near the center of the crossing, the system is described by a linear combination of the two states, but 
after the crossing, the system returns to one of the states. On the other hand, in the intermediate case, the system ends up being described by a linear combination of eigenstates. Similarly, when the external field is periodic, the time evolution depends on the way that avoided crossings between Floquet eigenstates (see Appendix A) are traversed. In Secs. II B and II C, we explain the differences between adiabatic and diabatic following for slow switching of Floquet Hamiltonians, after recalling in Sec. II A the main characteristics of adiabatic switching methods for generic Hamiltonians.

\section{A. Generic adiabatic switching}

The quantum adiabatic theorem $[10,11]$ states that, in the limit of infinitely slow passage of a Hamiltonian $H(t)$ from $t_{0}$ to $t_{f}$, the system will go from an initial state corresponding to an eigenstate of $H\left(t_{0}\right)$ to the instantaneous eigenstate of $H\left(t_{f}\right)$ continuously connected to it. The proof of the theorem requires the existence of a gap between the eigenvalue of interest and the rest of the instantaneous energy spectrum at all times. Physically, the slowness of the time variation of the perturbation is defined by a clock internal to the system, which is usually determined by the energy-level spacing and Planck's constant [12].

The adiabatic theorem is the basis of a strategy [13] for the calculation of excited eigenstates of time-independent Hamiltonians. Let us suppose that one is interested in the calculation of a given eigenstate of a Hamiltonian $H$ and that the eigenstates of another Hamiltonian $H_{0}$ are known. A virtual time-dependent Hamiltonian can be written down as

$$
H(t)=H_{0}+S(t) \Delta H,
$$

where

$$
\Delta H=H-H_{0},
$$

with $S(t)$ being a switching function that varies between 0 and 1 , for $0 \leqslant t \leqslant \tau$. In the limit $\tau \rightarrow \infty$ and for smooth enough switching functions, the adiabatic theorem guarantees that an eigenfunction of $H_{0}$ will evolve, at time $\tau$, to an eigenstate of $H$. This is illustrated in Fig. 1(b). The two curves represent two instantaneous eigenvalues that undergo an avoided crossing as a function of $S$, whose time dependence is represented in Fig. 1(a). If the variation of $S$ is slow enough, the evolving wave function is given at all times by the eigenstate whose associated eigenvalue is continuously connected to the initial state. Therefore, highly excited eigenfunctions of $H$ can be numerically obtained by solving the time-dependent Schrödinger equation for $H(t)$, without doing variational calculations [13]. Also, the method can be used in experiments to create excited states of $H$ starting from eigenstates of $H_{0}$, provided an appropriate Hamiltonian $H(t)$ can be realized in the laboratory.

\section{B. Adiabatic switching for Floquet Hamiltonians}

Adiabatic behavior of Floquet eigenstates is commonly invoked to describe time-dependent processes that involve a single laser pulse whose envelope varies slowly in time [14]. In this case, the period of the field is given by the inverse of the optical frequency of the laser. In particular, Floquet adiabatic switching has been applied to the calculation of properties

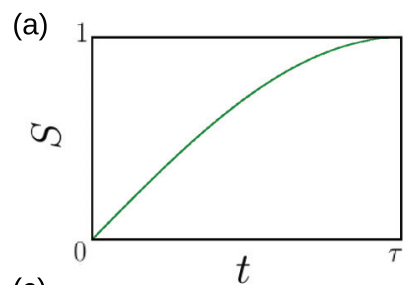

(c)

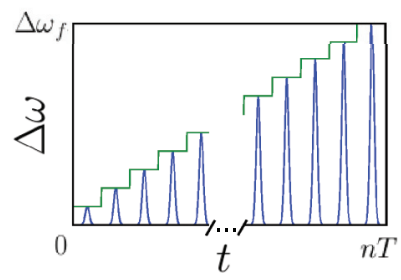

(b)

(d)

$\omega$

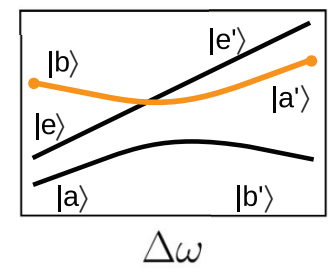

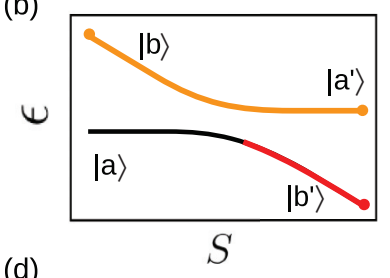

FIG. 1. (Color online) Illustration of switching strategies. (a) corresponds to Eq. (1) and shows the time variation of the switching function. The strict adiabatic limit corresponds to $\tau \rightarrow \infty$. However, approximate adiabaticity can be obtained for finite $\tau$. (b) shows the time dependence of two eigenvalues that undergo an avoided crossing. The orange (light gray) curve indicates the eigenvalue whose associated eigenstate contributes to the instantaneous time-evolved state when the evolution is adiabatic. The speed change of $S(t)$ needed to achieve adiabatic evolution is related to the size of the avoided crossing. When the evolution is not adiabatic, the wave function, if the crossing is traversed fully diabatically, is described by the red (dark gray) curve. (c) shows a stepwise variation of $\Delta \omega$ (see Appendix A) and the electric field for a pulse train. The adiabatic limit for Floquet eigenstates corresponds to $n \rightarrow \infty,\left[\Delta \omega_{n}-\Delta \omega_{n-1}\right] \rightarrow$ $0, \forall n$. (d) gives a pictorial representation of the two kinds of avoided crossings that can arise for quasienergy curves (see text).

for ionizing or dissociative Hamiltonians such as harmonic generation under intense laser radiation [15]. This approach is based on the use of Floquet theory combined with complex scaling, which is needed to isolate a resonance state from the other states within the continuum.

Here, we analyze if quantum adiabatic switching can be used to prepare selected Floquet eigenstates for a rigid rotor, which by definition is not dissociative. A fundamental difference with previous studies is that we consider a sequence of pulses instead of a single laser pulse, as shown in Fig. 1(c). In this case, the relevant frequency is the inverse of the repetition period between pulses, $\omega_{0}=2 \pi / T$, instead of the optical frequency because rapid laser oscillations at the optical frequency $\omega$ can be averaged out, as indicated in Appendix A.

Let us suppose that we would like to create a particular eigenstate for a given virtual Floquet Hamiltonian $\mathcal{F}_{f}$ [Eq. (A1)]. Specifically, we are interested in creating an aligned eigenstate for a Hamiltonian like that in Eq. (A3) for laser parameters $\Delta \omega_{f}, \sigma$, and $T$. If an adiabatic theorem holds and an eigenstate for an initial Floquet Hamiltonian $\mathcal{F}_{0}$ is available, an infinitely slow deformation of $\mathcal{F}_{0}$ up to $\mathcal{F}_{n}$ will take the initial Floquet eigenstate to the correlated Floquet eigenstate corresponding to $\mathcal{F}_{f}$.

Note that the global system is described by a timedependent Hamiltonian, Eq. (A3), where $\Delta \omega_{n}=S(t) \Delta \omega_{f}$, with $S(t)$ being a function that varies at times $t=t_{0}+n T$, $n=1,2, \ldots$. This global Hamiltonian is not periodic due to 
the variation of $\Delta \omega$. However, for each pulse we can define a virtual periodic system to which Floquet theory can be applied.

This strategy relies on the existence of an adiabatic theorem for Floquet Hamiltonians. The theorem has been proved only for Hilbert spaces of finite dimension [16]: For a system initially described by $\mathcal{F}_{0}$, if the time variation of $\Delta \omega_{n}$ is infinitely slow, the time-evolved state, when $n \rightarrow \infty$, converges to the Floquet state of the virtual periodic system corresponding to $\mathcal{F}_{f}$, found by continuously following the quasienergy of the initial state as a function of $\Delta \omega$. In practice, $\Delta \omega$ varies by discrete steps, but for small enough steps the evolution will be approximately adiabatic. The simplest choice for $\mathcal{F}_{0}$ corresponds to $\Delta \omega_{0}=0$. The eigenstates of the corresponding Floquet operator are field-free eigenstates.

Adiabatic switching of Floquet Hamiltonians, in a finite basis, is an unambiguous procedure in the sense that the correlation between initial and final states is established by quasienergy ordering of all levels pertaining to each symmetry species. Thus, at any given time, under adiabatic evolution, the progenitor state of a given state is clearly defined. If weak avoided crossings exist, the method can be difficult to implement since they must be traversed very slowly in order to follow quasienergy curves adiabatically.

\section{Diabatic switching for Floquet Hamiltonians}

Adiabatic following is not the only way to prepare Floquet eigenstates. In fact, as we will show for molecular alignment, it may not be the best option. If a large change in $\Delta \omega$ from one pulse to the next is chosen, diabatic crossings provide better continuation of eigenstates than adiabatic crossings [17]. We will show in Sec. III that such large increments in laser intensity may lead in some cases to fully diabatic crossings, resulting in the preparation of a single cyclic state. Before and after the crossing, the system is described by a single Floquet eigenstate. Thus, target cyclic states can be created using fewer pulses than with adiabatic following.

Relative populations of cyclic states in the final ensemble are different for diabatic and adiabatic following of avoided crossings since the progenitor state for each cyclic state is different in both cases. Under diabatic following, the correlation between states at two different field values is not determined by quasienergy ordering. In terms of ease of preparation, Hamiltonian parameters should be changed at the fastest possible rate so that molecules end up in a single Floquet eigenstate. Such a fast rate corresponds to diabatic traversing of avoided crossings.

The calculations shown in Sec. III indicate that a mixture of adiabatic and diabatic following, depending on the nature of the crossing, is the most convenient choice for the particular problem of molecular alignment. The relevance of different kinds of avoided crossings can be understood by examining Fig. 1. As shown in Fig. 1(d), a given quasienergy curve can present weak avoided crossings with curves coming from highly excited field-free states and strong avoided crossings with curves belonging to quasienergy states with similar rotational compositions. These crossings can take place in succession or simultaneously, such that many avoided crossings of both types can occur during the time evolution of a particular initial state. State $|b\rangle$, in Fig. 1(d), starts as a low- $J$ eigenstate that gets progressively aligned when the field increases. State $|a\rangle$ is correlated to an initial state with higher $J$ that is not aligned. Since the two states interchange character at the crossing, the state $\left|a^{\prime}\right\rangle$ adiabatically correlated to $|b\rangle$ is not aligned. Adiabatic traversing of the crossing implies that molecules in state $|b\rangle$ get misaligned after the crossing. Also, sporadic and numerous crossings with highly excited rotational states can take place. In the theoretical limit $J \rightarrow \infty$ (see Appendix B) these crossings occur infinitely often. In Fig. 1, for simplicity, we have plotted only one of these states, $|e\rangle$. Frequent avoided crossings between states with low and very high $J$ are due to high-order resonances and are ineffective and will be traversed diabatically. For all practical purposes, these avoided crossings behave like true crossings since they do not alter the time evolution calculated within a small basis set.

Properties of avoided crossings for Floquet eigenstates were studied in Refs. [18,19]. For the driven square, two kinds of crossings, sharp and broad, were identified by Timberlake and Reichl [18]. These authors studied the influence of the type of crossing on the structure of the resulting Floquet states. While sharp crossings lead to temporary changes as a function of field strength, broad crossings can give rise to delocalized states. These changes affect the strength of high-harmonic radiation [18]. Qualitatively similar changes arise in the context of molecular alignment.

\section{PREPARATION OF CYCLIC ROTATIONAL STATES}

Here, we discuss optimal strategies for preparing cyclic rotational states. Reduced units for time and energy are used in the calculations as explained in Appendix A. These units depend on the rotational constant $B$, and therefore, our results can be extrapolated to any linear molecule.

For an external field with linear polarization, the Floquet matrix contains nonzero elements between states with the same magnetic quantum number $M$ due to the selection rule $\Delta M=$ 0 . Thus, the matrix is divided into independent blocks, one for each $M$ value. Matrices for positive and negative $M$ are identical, so only one needs to be calculated. Another selection rule is $\Delta J=0, \pm 2$. Thus, the different $M$ matrices are, in turn, divided into two independent blocks, one for even $J$ and another one for odd $J$.

In the following we will frequently refer to rotational states as low- $J$ or high- $J$ states. In general, it will be implied that low- $J$ states are those for which $J<10$. This follows from the observation that optimum alignment in the pendular limit is already obtained for wave packets formed by a linear combination, with the appropriate phases, of rotational eigenstates with $J<10$.

As described in Appendix B, Floquet spectra for rotational Hamiltonians are dense in the theoretical limit $J \rightarrow \infty$. This limit cannot be reached in real systems, but even so, a large number of rotational basis functions must be included in the Floquet matrix to obtain converged quasienergies. In general, the size of the matrix increases with the field strength. On the other hand, the size of the time Fourier basis set needed for achieving convergence increases with the period of the pulse train. 

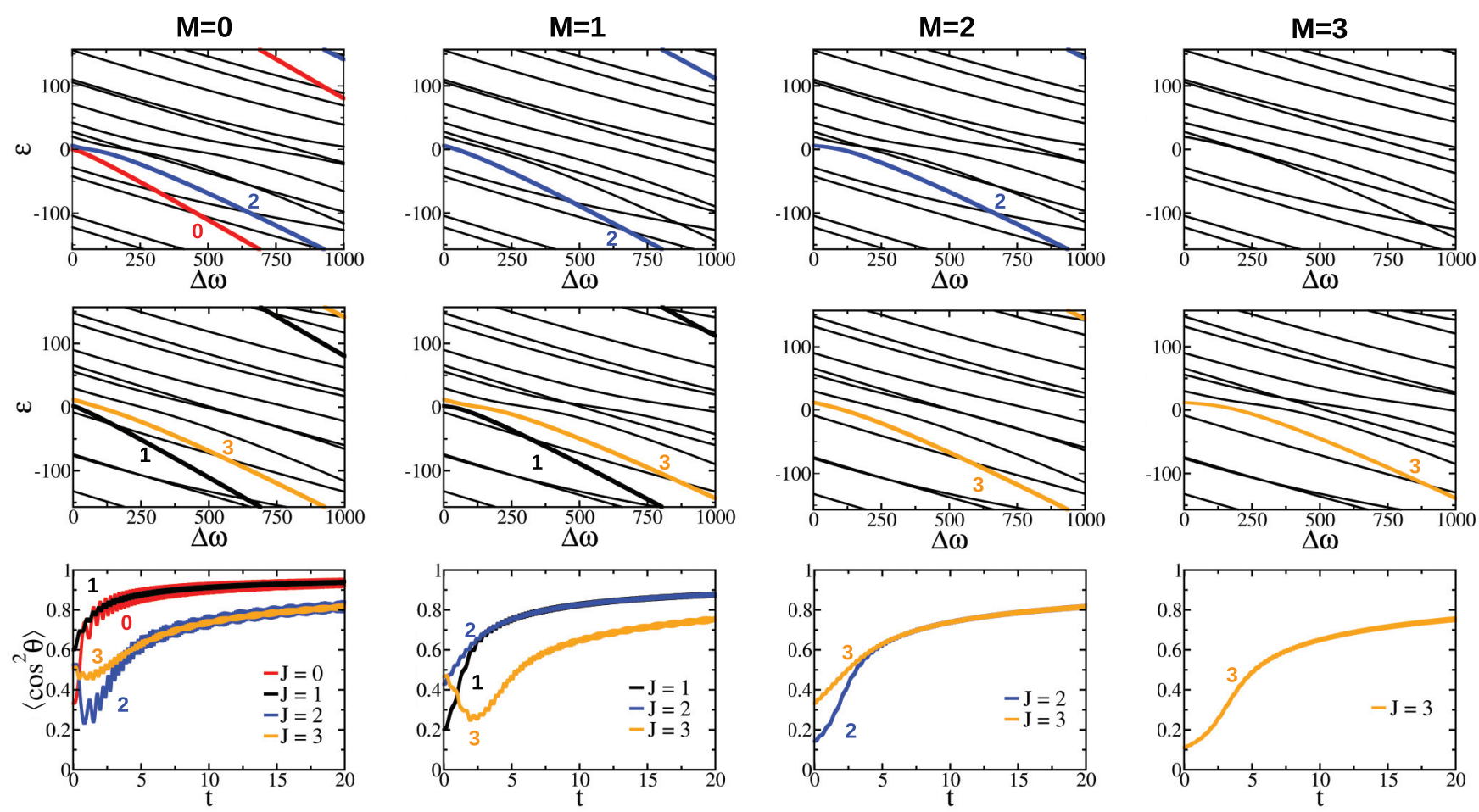

FIG. 2. (Color online) The top row shows quasienergies $\varepsilon$, in units of $B / \hbar$, within the first Brillouin zone (see Appendix A) for quasienergy states with initial quantum number $M \leqslant 3$ and even $J$ as a function of $\Delta \omega$ (dimensionless) for a train with pulse parameters $\sigma=0.003 \hbar / B$ and $T=0.02 \hbar / B$. The middle row shows the same for odd $J$. The bottom row shows the evolution of the average instantaneous alignment $\left\langle\cos ^{2} \theta\right\rangle$ as a function of time $t$, in units of $(\hbar / B)$, for ten initial states, as indicated in the plots. The color scale (gray scale) in the curves for the quasienergy plots indicates the quasienergy state associated with each alignment curve. A fixed field step such that the increment in $\Delta \omega$ is 1 has been chosen for all the calculations.

First, we study a case corresponding to a train composed of Gaussian pulses with a repetition period between consecutive pulses much shorter than a rotational period, i.e., $T<\pi / 100$. Figure 2 shows quasienergies, within the first Brillouin zone, as a function of the field amplitude. For the range of $\Delta \omega$ values used in our calculations, a basis set containing rotational states up to $J=25$ is enough to obtain convergence for the time evolution of initial low- $J$ field-free rotational eigenstates. For zero field, the quasienergy spectrum is given by the field-free energy spectrum modulo $\omega_{0}$. When the field increases, a growing interaction between quasienergy states takes place in an ordered way. Due to the quadratic dependence on $J$ of rotational energies, interactions between low- $J$ states are strong, while high- $J$ states are less affected. Note that unlike the normal situation for spatial Hamiltonians, quasidegenerate Floquet eigenstates can have a very different rotational composition, resulting in weak interactions.

The main characteristics of quasienergy curves do not change much with the magnetic quantum number $M$. There are a few sharp avoided crossings that, at the scale shown in Fig. 2, appear like true crossings. This implies that the interaction between the two states involved in each of these crossings is very small. Some states are quasidegenerate for a large range of field strengths, although for the strongest field shown in Fig. 2 the degeneracies disappear. For low fields, a few strong avoided crossings take place.
Due to the small dimension of the effective rotational Hilbert space in the short-pulse regime, it is appropriate to change the field strength from one pulse to the next by a fixed amount. The bottom row in Fig. 2 shows instantaneous alignment for all initial states with $J \leqslant 3$. Excellent alignment is obtained in all cases with realistic fields, i.e., fields that are weak enough to avoid photodissociation of the molecules. Except in two cases, our calculations show that, under the chosen conditions, we obtain a single cyclic state for the last pulse. This implies that all the crossings have been traversed either fully diabatically or fully adiabatically. The two exceptions correspond to initial states $|J=0, M=0\rangle$ and $|J=2, M=0\rangle$. In these two cases, the initial state gets mixed with another state due to the existence of a repulsive avoided crossing for $\Delta \omega \approx 25$. In spite of the mixture with a second Floquet eigenstate, excellent alignment is achieved in these cases too, although rapid alignment oscillations take place. These oscillations are due to the difference in quasienergies between the two Floquet eigenstates that form the wave packet.

On the other hand, the use of a smaller field step leads to a single cyclic state for the two exceptional states, as shown in Fig. 3. This figure shows the instantaneous average alignment during a sequence of 55 identical pulses with $\Delta \omega=135.25$, $\sigma=0.003 \hbar / B$, and $T=0.02 \hbar / B$ for two different initial states. These two states result from submitting the state $|J=0, M=0\rangle$ to a pulse train with the same $\sigma, T$ and 


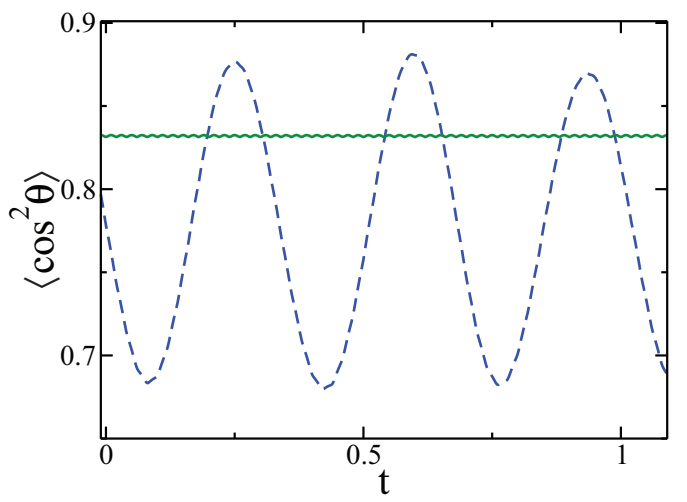

FIG. 3. (Color online) Instantaneous average alignment during a sequence of laser pulses with $\sigma=0.003 \hbar / B, T=0.02 \hbar / B$, and $\Delta \omega=135.25$. Time $t$ is in units of $\hbar / B$. The initial state is a single Floquet eigenstate (solid line) or a linear combination of two Floquet states (dashed line). These two initial rotational wave packets were obtained by increasing the field strength during a pulse sequence. The single Floquet state was obtained using $\Delta \omega$ steps of size 0.25 . The mixed state was obtained when $\Delta \omega$ changed by steps of 2.5 . For the mixed state, alignment oscillates during the subsequent pulse train. However, for the single Floquet eigenstate the alignment is quasiconserved.

increasing intensity. One state is obtained by using $\Delta \omega$ steps of 0.25 , and the other one is obtained by using steps of 2.5 .

The time evolution for initial low- $J$ states does not change when the basis set increases. Note that this would not be so under the strict adiabatic limit, $\left(\Delta \omega_{n}-\Delta \omega_{n-1}\right) \rightarrow 0, \forall n$. Assuming that this limit could be implemented in practice, the evolved states would depend on the highest excited $|J, M\rangle$ state included in the basis set because in the adiabatic limit all avoided crossings, even those considered to be ineffective, would be adiabatically traversed. Thus, strict adiabatic time evolution would take molecules from field-free low- $J$ states to high- $J$ cyclic states, which will hardly show any alignment. In conclusion, the appropriate strategy for aligning a molecular ensemble requires diabatic following at every weak crossing.

Larger rotational basis sets are needed to converge high- $J$ levels. This leads to a complex network of avoided crossings. However, crossings between states converged within the smaller basis and high- $J$ states, converged with the larger basis, are inefficient. Therefore, they are traversed diabatically even for exceedingly small field steps.

\section{MISALIGNMENT EFFECTS}

\section{A. Effects due to strong avoided crossings}

We have shown that aligned cyclic states can be created from a field-free ensemble of rotational eigenstates by slowly switching the field strength during a train composed of pulses much shorter than the rotational period.

When a single nonresonant laser pulse is used, the value of $\left\langle\cos ^{2} \theta\right\rangle$, for a given $|M|$, moves from its high-field limit to its free-rotor limit as $J$ increases [20]. The reason for this behavior is that molecules become aligned when they are bound within an effective potential that determines the range in which the molecular axis can librate. In the oblate spheroidal case, to which all linear molecules belong, this effective potential [20] can be written as

$$
V_{\mathrm{eff}}=\left(\frac{M^{2}-1 / 4}{\sin ^{2} \theta}-\frac{1}{4}\right)-\Delta \omega \cos ^{2} \theta .
$$

$V_{\text {eff }}$ has two minima, whose locations depend on $|M|$ and $\Delta \omega$. States with smaller $J-|M|$ get bound first, while states with higher $J$ for a given $|M|$ require stronger fields to reach the pendular limit, $\left\langle\cos ^{2} \theta\right\rangle \rightarrow 1$. A similar trend, except for initial transients, occurs for trains composed of short pulses, as shown in Fig. 2. The initial transients are due to the fieldfree value of $\left\langle\cos ^{2} \theta\right\rangle$, which can be greater for states with higher $J$.

For trains with longer pulses or longer pulse delay, the situation is more complex. In these cases and for strong fields, avoided crossings take place between pairs of states adiabatically correlated to initial low- $J$ states. The effect of these crossings can be understood by examining Fig. 4. The time dependence of alignment for the initial state $|J=1, M=0\rangle$ is shown for two similar pulses with slightly different $\sigma$ and $T$ values. For the longer pulse, the alignment is eventually lost due to the presence of an avoided crossing, near $\Delta \omega \approx 135$, with a state whose progenitor state is $|J=5, M=0\rangle$. For the chosen field step, the evolution is adiabatic, and the molecule is described after the crossing by a single cyclic state. However, this cyclic state is less aligned than the cyclic state before the crossing due to the well-known fact that states interchange character at avoided crossings [21]. For pulses with $\sigma=0.16 \hbar / B$ the cyclic state correlated to the initial state $|J=1, M=0\rangle$ does not show any crossing, and the alignment steadily increases with the field intensity. On the other hand, the initial state $|J=0, M=0\rangle$ features up to four weak avoided crossings with excited states, but no strong avoided crossings take place. The weak avoided crossings are diabatically traversed, giving rise to increasing alignment.

In general, low- $J$ levels need weaker fields than high- $J$ levels to become well aligned. However, the phenomenon analyzed in Fig. 4 indicates that alignment of an ensemble that contains molecules with different $J$ quantum numbers is not a matter of just increasing the field. In effect, if we increase the field strength to align excited rotational states, avoided crossings involving these states with low- $J$ states, which became aligned with weaker fields, take place. These crossings cannot be traversed diabatically. Thus, in order to stay in a single cyclic state, they must be crossed adiabatically. As a result, the state with the lower $J$ value gets misaligned after the crossing. In other words, although excited states may be aligned with stronger fields, low- $J$ states undergo highfield avoided crossings, becoming simultaneously misaligned. Thus, molecular ensemble alignment requires a trade-off in the field strength to align excited states without misaligning states with lower $J$.

This phenomenon is fairly robust with respect to the laser duration, but it is more fragile with respect to the time delay between pulses. This is due to the fact that the exact location of strong avoided crossings is more sensitive to the temporal spacing between the laser pulses than to the time width of each pulse. Thus, small changes in the parameter $T$ alters the time evolution. This phenomenon can be exploited to design appropriate fields. Optimal ensemble alignment 

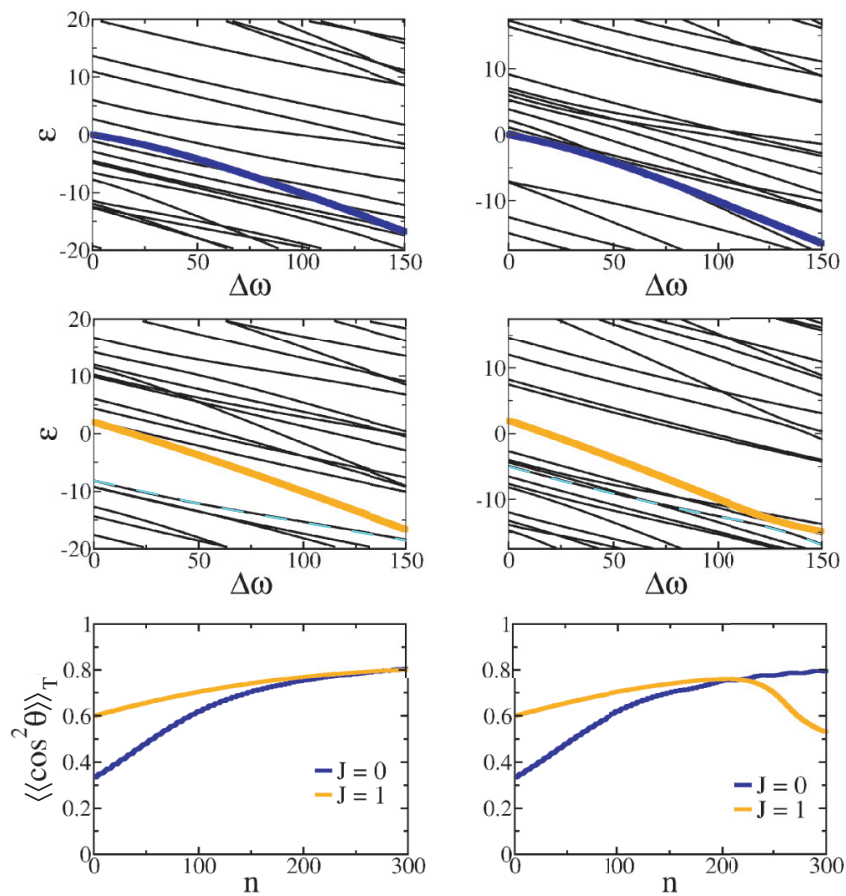

FIG. 4. (Color online) Sensitivity of avoided crossings to the period of the laser pulses. Top and middle rows show quasienergies $\varepsilon$, in units of $B / \hbar$, as a function of $\Delta \omega$ (dimensionless) within the first Brillouin zone and average alignment $\left\langle\left\langle\cos ^{2} \theta\right\rangle\right\rangle_{T}$ during each pulse for two pulse sequences, with $\sigma=T / 10$. Plots in the left column are for $T=0.16 \hbar / B$, and plots in the right column are for $T=0.18 \hbar / B$. Plots in the top row show quasienergy curves for even $J$. Plots in the middle row correspond to odd $J$. Alignment as a function of pulse number $n$ is shown in the bottom row. Alignment of the initial state $|J=0, M=0\rangle$ is similar for both cases. However, the state $|J=1, M=0\rangle$ loses the alignment for the longer pulse due to the existence of a strong avoided crossing near $\Delta \omega=135$ with a state whose progenitor state is $|J=5, M=0\rangle$ (dashed curve in the corresponding quasienergy plot). For a step of 0.5 in $\Delta \omega$, the crossing is adiabatically traversed. Diabatic traversing of the crossing would avoid the misalignment. However, due to the accidental degeneracy between the two states, larger field steps do not lead to pure diabatic crossing but to a mixture of the two states involved in the crossings, with the subsequent loss of alignment. For the shorter pulse, the initial state $|J=1, M=0\rangle$ does not show any crossings, although it is approaching one with the highly excited state $|J=17, M=0\rangle$ (dashed curve in the corresponding quasienergy plot).

requires designing pulse trains especially tailored to navigate through the net of avoided crossings.

\section{B. Effects due to accidental degeneracies}

Accidental quasidegeneracies between quasienergy states can arise even within a small rotational basis set. The interaction between these quasidegenerate states depends basically on the $T$ value. Figure 5 shows quasienergy levels for a pulse train with $T=0.3 \hbar / B$. Quasienergy states coming from initial states $|J=0, M=0\rangle$ and $|J=1, M=0\rangle$ exhibit quasidegeneracies with other states near $\Delta \omega=0$. The state quasidegenerate with $|J=1, M=0\rangle$ is $|J=17, M=0\rangle$, and the interaction between them is not efficient. Thus, this state
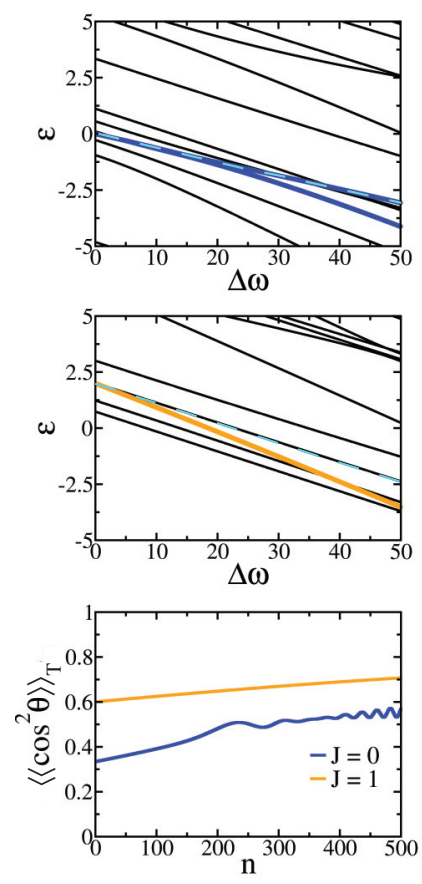

FIG. 5. (Color online) Quasienergies $\varepsilon$, in units of $B / \hbar$, as a function of dimensionless $\Delta \omega$ (top and middle panels) and average alignment $\left\langle\left\langle\cos ^{2} \theta\right\rangle\right\rangle_{T}$ (bottom panel) during a laser period as a function of pulse number $n$ for a pulse train with $\sigma=0.03 \hbar / B$ and $T=0.3 \hbar / B$. The rotational ground state $|J=0, M=0\rangle$ ends up less aligned than the initial state $|J=1, M=0\rangle$. The ground state is nearly degenerate with $|J=6, M=0\rangle$ (dashed curve in the quasienergy plot shown in the top panel) for a large range of $\Delta \omega$ values. Thus, diabatic crossing is not possible. Adiabatic following is theoretically possible but requires a very small field step. A wave packet formed by a linear combination of both states develops, which gives rise to oscillations in the alignment. The state $|J=1, M=0\rangle$ is nearly degenerate, near $\Delta \omega=0$, with the excited state $|J=17, M=0\rangle$ (dashed curve in the quasienergy plot shown in the middle panel). The interaction between both states is negligible, and $|J=1, M=0\rangle$ gets aligned when the field increases. The time evolution has been calculated using $\Delta \omega$ steps of 0.1 .

becomes better aligned than the rotational ground state, which interacts more strongly with the state correlated to the field-free state $|J=6, M=0\rangle$. Contrary to the avoided crossings that arise for shorter pulses at intense fields, adiabatic following is difficult in the presence of interacting quasidegenerate states and requires very small field steps.

This example gives an indication of the rich dynamics that takes place due to the complex structure of quasienergy spectra. This structure can be exploited to create specific aligned states that otherwise would be difficult to prepare.

In practice, a general strategy to avoid misalignment effects is to use, for weak fields, an optimized pulse train that must vary slowly enough to avoid mixing neighbor cyclic states. Once pendular states are created, the field can be increased faster to diabatically cross further weaker resonances. However, when the condition $T \leqslant 0.1$ does not hold, repulsive crossings between low- $J$ states arise before maximum alignment is reached for most states. The consequence is that the maximum ensemble alignment is limited. 


\section{CONCLUSIONS}

We have presented a strategy to create aligned cyclic wave packets for molecular ensembles. The method is based on slowly switching the field amplitude during a sequence of nonresonant laser pulses from zero to a selected target field. The appropriate strategy depends on the duration of the pulse repetition period compared to the molecule rotational period. For a short delay between pulses no strong avoided crossings take place before the states get aligned. Thus, large enough field steps lead to diabatic following of Floquet eigenstates, and single cyclic states are prepared in a straightforward way. These states can be further submitted to a periodic sequence of identical pulses to maintain long-lasting alignment.

High- $J$ states are relatively unaffected by laser intensity. Their corresponding quasienergy curves decrease slowly, and basically, they remain parallel. Eventually, low- $J$ quasienergy states interact with higher- $J$ states due to high-order resonances. For short pulses these resonances are not efficient except at very high fields because the rotational compositions of the states involved are quite different. As a result, these weakly repulsive avoided crossings can be crossed diabatically, as required to keep the time-evolved state well aligned. On the other hand, adiabatic following of such weak crossings would destroy the alignment, transforming the evolving cyclic state into a misaligned high- $J$ cyclic wave packet.

When the dimension of the effective rotational Hilbert space is small, it is appropriate to change the field strength from one pulse to the next by a fixed amount. This technique is useful when all crossings are equally efficient. However, when the number of rotational states needed to achieve convergence increases, the structure of the avoided crossing net is not uniform anymore, and variable step sizes must be employed. As a general rule, for the first pulses of the train, that is, at low fields, the variation must be slow to avoid oscillations in the alignment. When the intensity of the field increases, new avoided crossings must be traversed diabatically to avoid mixing with other states. This requires a faster variation of the field.

For fields that are strong enough, pure diabatic following is impossible to achieve due to the presence of strong avoided crossings. Therefore, there is a limit in the field strength that can be used to implement the method. This limit restricts the highest excited rotational state that can be converted into a cyclic state without simultaneously misaligning low- $J$ states. For all practical purposes, a good alignment strategy is achieved by choosing the repetition period of the pulse train such that maximum alignment is obtained for several $J$ states before resonant avoided crossings become too strong. This situation is reached for $T \leqslant 0.1 \hbar / B$ and $0.02 \leqslant \sigma / T \leqslant$ 0.16 , which implies a limit on the kinds of molecules to which the method can be applied. For example, for $\mathrm{N}_{2}(B \approx$ $2 \mathrm{~cm}^{-1}$ ) excellent alignment can be achieved with $T \leqslant$ $250 \mathrm{fs}$ and $\sigma$ between 5 and $40 \mathrm{fs}$. On the other hand, for $\mathrm{H}_{2}\left(B \approx 60.8 \mathrm{~cm}^{-1}\right)$, implementation of the method would require subfemtosecond pulses.

From the experimental viewpoint it is important to limit the maximum laser power in order to avoid molecular ionization. For a given $\Delta \omega$ the laser power is inversely related to the quotient between molecular polarizability and the rotational constant. For example, $\Delta \omega=1000$ is equivalent to a laser power of approximately $0.9 \times 10^{14} \mathrm{~W} / \mathrm{cm}^{2}$ for $\mathrm{N}_{2}$ but of only $3 \times 10^{11} \mathrm{~W} / \mathrm{cm}^{2}$ for $\mathrm{I}_{2}$. This shows that the method is especially well suited for highly polarizable heavy molecules for which trains with a delay between pulses of tens of picoseconds can be used with peak powers well below the limit for which molecules will photodissociate.

Regarding experimental implementations of our method, it must be taken into account that pulse trains can be generated by recently developed pulse-shaping techniques [22,23]. The key idea is to synthesize the train by phase control and locking of many sidebands with $\omega_{0}$ frequency spacing. These sidebands are generated from an initial strong laser pulse whose optical frequency can be chosen to avoid unwanted resonances for the molecule of interest.

Parameters in the Floquet Hamiltonian other than the pulse intensity can be slowly switched. In the present work we have analyzed the method for pulses that have varying strength. Alternatively, the intensity can be fixed, and the time width of the laser can be varied, or intensity and width can be kept constant for all pulses in the train, and the delay between pulses can be varied. Finally, additional fields can introduce new control parameters, which may be tuned for the design of different circuits in the parameter space.

\section{ACKNOWLEDGMENT}

Financial support from the Spanish government through MICINN Project No. FIS2010-18799 is acknowledged.

\section{APPENDIX A: ROTATIONAL CYCLIC STATES}

A time-dependent periodic system can be transformed into a time-independent one represented by an infinite matrix [24]. Sambe [25] showed that, by treating time as another spatial coordinate, quasienergy states, conceptually equivalent to the stationary states of conservative quantum systems, can be defined. These steady states $\lambda$ are the eigenstates of the Floquet Hamiltonian,

$$
\mathcal{F} \equiv-i \hbar \frac{\partial}{\partial t}+H(t),
$$

where $H(t)$ is the usual spatial Hamiltonian plus an external perturbation that depends explicitly on time. Eigenstates of $\mathcal{F}$ belong to an extended Hilbert space formed by the composition of the Hilbert space corresponding to the spatial part of the Hamiltonian operator and the space formed by all possible periodic Fourier basis functions of the time coordinate, with finite norm [26]. Yajima [27] and Howland [28] showed that for periodic systems, the so-called Floquet operator, that is, the time-evolution operator during one period $T$ of the external perturbation $U\left(T+t_{0}, t_{0}\right)$, and the Floquet Hamiltonian are spectrally equivalent. Thus, the eigenstates of the Floquet Hamiltonian are cyclic since the following equation holds for the Floquet operator:

$$
U\left(T+t_{0}, t_{0}\right)\left|\lambda\left(t_{0}\right)\right\rangle=e^{-(i / \hbar) \varepsilon T}\left|\lambda\left(t_{0}\right)\right\rangle,
$$

where $\varepsilon$ is the eigenvalue associated with the eigenstate $\lambda$ of $\mathcal{F}$.

The Hamiltonian, in dimensionless form (which suggests using $\hbar / B$ as a reduced unit of time and $B / \hbar$ as a reduced 
unit of frequency, with $B$ being the rotational constant of the molecule), for a rigid linear molecule in the presence of a pulse train composed of linearly polarized nonresonant Gaussian laser pulses is $[3,4]$

$$
H(t)=\mathbf{J}^{2}-\sum_{n} \frac{\Delta \alpha E_{n}^{2}}{2 B} \exp \left[\frac{-(t-n T / 2)^{2}}{\sigma^{2}}\right] \cos ^{2} \theta \cos ^{2}(\omega t),
$$

where $\mathbf{J}$ is the angular momentum operator and $\sigma$ gives the time width of the laser. The angle $\theta$ is the polar angle between the internuclear axis and the field direction. The constant $\Delta \alpha$ is the polarizability anisotropy, and $E_{n}$ is the strength of the electric field for pulse $n$. In the previous expression a term coming from the perpendicular component of the polarizability $\alpha_{\perp}$ has been neglected since it only adds a constant energy shift. The interaction of a permanent dipole with a rapidly oscillating field averages to zero. In the same way, the term $\cos ^{2}(\omega t)$ in Eq. (A3) can be averaged out, which gives an extra factor, 1/2, which is usually included in the definition of the parameter $\Delta \omega=\Delta \alpha E^{2} /(4 B)$. Averaging out the optical frequency is equivalent to considering an external field given by the squared envelope of the laser field. The relevant frequency is then given by the repetition frequency of the pulse train, i.e., $\omega_{0}=2 \pi / T$. The dimensionless parameter $\Delta \omega$ is related to the polarizability, laser intensity, and rotational constant by

$$
\Delta \omega=10^{-11} \Delta \alpha\left(\AA^{3}\right) I\left(\mathrm{~W} / \mathrm{cm}^{2}\right) / B\left(\mathrm{~cm}^{-1}\right) .
$$

The time evolution for an initial ket $\left|\psi\left(t_{0}\right)\right\rangle$ during a periodic pulse train, that is, when $\Delta \omega$ remains constant, can be calculated within the Floquet approach, in reduced units, from the expression [29]

$$
|\psi(t)\rangle=\sum_{j} \exp \left[-i \varepsilon_{j}\left(t-t_{0}\right)\right]\left|\lambda_{j}(t)\right\rangle\left\langle\lambda_{j}\left(t_{0}\right) \mid \psi\left(t_{0}\right)\right\rangle,
$$

where the index $j$ runs over states whose quasienergies belong to the first Brillouin zone, i.e., $\varepsilon_{j} \in[-\pi / T, \pi / T]$. Equation (A5) is restricted to a single Brillouin zone due to the periodicity of the system. The number of Floquet eigenvalues inside a Brillouin zone is equal to the number of spatial basis functions used to build the Floquet matrix.

The time evolution during a sequence of pulses when the laser intensity changes from pulse to pulse can be calculated in an equivalent way. For that, it is assumed that each pulse belongs to a virtual periodic pulse train. Thus, the wave function during pulse $n$ is given by Eq. (A5) if $\left|\psi\left(t_{0}\right)\right\rangle$ is taken as the evolved wave function at the end of pulse $n-1$. Thus, the wave function during the time interval $n T-T / 2 \leqslant$ $t \leqslant n T+T / 2$ is

$$
\begin{aligned}
|\psi(t)\rangle= & \sum_{j} \exp \left[-i \varepsilon_{j}^{n}(t-n T+T / 2)\right]\left|\lambda_{j}^{n}(t)\right\rangle \\
& \times\left\langle\lambda_{j}^{(n-1)}(n T-T / 2) \mid \psi(n T-T / 2)\right\rangle .
\end{aligned}
$$

Under adiabatic conditions the time-evolved wave function when the initial state is a rotational eigenstate, i.e., $|\psi(-T / 2)\rangle=\left|\lambda_{1}^{0}(-T / 2)\right\rangle=|J, M\rangle$, becomes for pulse $n$

$$
|\psi(t)\rangle \approx \exp (-i \delta)\left|\lambda_{1}^{n}(t)\right\rangle
$$

where

$$
\delta=\sum_{j=1}^{n} \varepsilon_{1}^{j}(t-j T+T / 2)
$$

\section{APPENDIX B: UNBOUNDED ROTATIONAL SPECTRA}

Rotational Floquet Hamiltonians have dense spectra in the theoretical limit $J \rightarrow \infty$. Several conclusions can be obtained from the analysis of this limit whenever a given model requires us to include a large number of rotational states. As a function of a parameter in the Hamiltonian, quasienergy curves exhibit infinitely many avoided crossings in any quasienergy interval $[16,30]$. It has been conjectured that, in such a limit, an adiabatic theorem for Floquet Hamiltonians does not hold [16], although special versions of the adiabatic theorem have been demonstrated [12] for generic Hamiltonians (but not for Floquet Hamiltonians) in the absence of spectral gaps.

Technically, two different cases may exist for Floquet Hamiltonians [31]: (i) The spectrum of $\mathcal{F}$, Eq. (A1), is singular continuous at least for some $\Delta \omega$ values. In this case, quasienergies are discontinuous functions of $\Delta \omega$, and the spectrum present holes [16]. When the variation of $\Delta \omega$ is infinitely slow, the system necessarily runs into these holes, and an adiabatic theorem does not hold (Proposition II of Ref. [16]). In this case, it is still possible that the system evolves to a state $\lambda$ which is an approximate solution to $(\mathcal{F}-\epsilon) \lambda \approx 0$ [32]. (ii) The spectrum of $\mathcal{F}$, although dense, is pure point; that is, eigenstates exist for all $\Delta \omega$ values. In this case, a truncated matrix representation of $\mathcal{F}$ gives quasienergies within an error that can be made as small as desired by increasing the matrix size (Proposition I of Ref. [16]).

Let us suppose that the variation of $\Delta \omega$ depends on the pulse number $n$ and on a parameter $s$ through a function $S \propto(1-$ $\left.e^{-s n}\right)$ [16]. The adiabatic theorem for Floquet Hamiltonians with a finite number of states $J_{\max }$ indicates that for a particular truncation there is an $s_{\mathrm{a}}$ value such that for $s \leqslant s_{\mathrm{a}}$ all the avoided crossings are adiabatically traversed. For a truncated matrix with $J_{\max }^{\prime}>J_{\max }$, new avoided crossings take place. For these new crossings, one of the states does not exist for the truncation $J_{\max }$.

Assuming that new crossings, involving at least one low- $J$ state, are less effective than those already present for the $J_{\max }$ truncation, there may be an $s_{M}$ value, with $s_{M} \leqslant s_{\mathrm{a}}$, such that for $s \geqslant s_{M}$ all new crossings are diabatically traversed. Then, there is a window of $s$ values, $\left[s_{M}, s_{\mathrm{a}}\right]$, for which adiabatic evolution of initial cyclic states, with $J \ll J_{\max }$, of a Floquet matrix of size $J_{\max }$ faithfully represents the time evolution of cyclic states of a representation with $J_{\max }^{\prime}>J_{\max }$. This behavior can be extended to the $J_{\max }^{\prime} \rightarrow \infty$ limit, and it is a pedestrian version of the ineffectiveness of high-order resonances in which adiabatic theorems for systems with a dense spectrum are founded [12]. If, for a particular size $J_{\max }^{\prime}$, $s_{M}>s_{\mathrm{a}}$, adiabatic time evolution within the truncated Hilbert space of size $J_{\max }$ does not represent the actual evolution in the Hilbert space of size $J_{\max }^{\prime}$. For rotational Hamiltonians interacting with linearly polarized lasers (selection rule $\Delta M=$ 0 ), due to the increasing spacing between energy levels with $J$, this situation never occurs. 
For a finite $J_{\max }$, adiabatic following of Floquet eigenstates is not the preferred method for ensemble alignment when there are weak but effective avoided crossings. Fully diabatic following is more economical in terms of the number of pulses needed to prepare a particular cyclic state. Therefore, if for a given $J_{\max }$ truncation of the Floquet Hamiltonian, there is an $s_{\mathrm{d}}$ value such that all avoided crossings are diabatically traversed, new crossings arising for a larger truncation, $J_{\max }^{\prime}$, will be diabatically traversed too for $s=s_{\mathrm{d}}$. Then, diabatic following for all avoided crossings between cyclic states corresponding to a Floquet matrix of size $J_{\max }$ faithfully represents the actual time evolution for $J_{\max }^{\prime}$.

Finally, it should be stressed that since the spectrum is dense, diabatic following takes low- $J$ field-free eigenstates to aligned cyclic eigenstates. However, adiabatic following of all avoided crossings would take molecules from field-free eigenstates to states whose quasienergy is arbitrarily close to the initial field-free energy [16] and that therefore are misaligned.
[1] H. Stapelfeldt and T. Seideman, Rev. Mod. Phys. 75, 543 (2003).

[2] B. Friedrich and D. R. Herschbach, Z. Phys. D 18, 153 (1991).

[3] J. Ortigoso, M. Rodríguez, M. Gupta, and B. Friedrich, J. Chem. Phys. 110, 3870 (1999).

[4] J. Ortigoso, Phys. Rev. Lett. 93, 073001 (2004).

[5] R. S. Judson, K. K. Lehmann, H. Rabitz, and W. S. Warren, J. Mol. Struct. 223, 425 (1990); J. Ortigoso, Phys. Rev. A 57, 4592 (1998).

[6] J. G. Underwood, M. Spanner, M. Y. Ivanov, J. Mottershead, B. J. Sussman, and A. Stolow, Phys. Rev. Lett. 90, 223001 (2003).

[7] J. Ortigoso and J. Santos, Phys. Rev. A 72, 053401 (2005).

[8] E. A. Shapiro, V. Milner, C. Menzel-Jones, and M. Shapiro, Phys. Rev. Lett. 99, 033002 (2007).

[9] W. Zhao, J. J. Mestayer, J. C. Lancaster, F. B. Dunning, C. O. Reinhold, S. Yoshida, and J. Burgdörfer, Phys. Rev. Lett. 97, 253003 (2006); T. Kopyciuk and R. Parzyński, Phys. Rev. A 75, 055402 (2007); C. O. Reinhold, S. Yoshida, J. Burgdörfer, B. E. Tannian, C. L. Stokely, and F. B. Dunning, J. Phys. B 34, L551 (2001); S. Yoshida, C. O. Reinhold, E. Persson, J. Burgdörfer, and F. B. Dunning, ibid. 38, S209 (2005).

[10] A. Messiah, Quantum Mechanics (North-Holland, Amsterdam, 1962), Vol. 2.

[11] J. Ortigoso, Phys. Rev. A 86, 032121 (2012).

[12] J. E. Avron, J. S. Howland, and B. Simon, Commun. Math. Phys. 128, 497 (1990).

[13] D. Kohen and D. J. Tannor, J. Chem. Phys. 98, 3178 (1993).
[14] K. Drese and M. Holthaus, Eur. Phys. J. D 5, 119 (1999).

[15] N. Ben-Tal, N. Moiseyev, and R. Kosloff, Phys. Rev. A 48, 2437 (1993).

[16] D. W. Hone, R. Ketzmerick, and W. Kohn, Phys. Rev. A 56, 4045 (1997); Phys. Rev. E 79, 051129 (2009).

[17] R. T. Skodje and F. Borondo, J. Chem. Phys. 85, 2760 (1986).

[18] T. Timberlake and L. E. Reichl, Phys. Rev. A 59, 2886 (1999).

[19] B. P. Holder and L. E. Reichl, Phys. Rev. A 72, 043408 (2005).

[20] B. Friedrich and D. Herschbach, J. Phys. Chem. 99, 15686 (1995).

[21] K. Na and L. E. Reichl, Phys. Rev. A 70, 063405 (2004).

[22] A. V. Sokolov and S. E. Harris, J. Opt. B 5, R1 (2003).

[23] K. R. Pandiri and M. Katsuragawa, New. J. Phys. 13, 023030 (2011).

[24] J. H. Shirley, Phys. Rev. 138, B979 (1965).

[25] H. Sambe, Phys. Rev. A 7, 2203 (1973).

[26] U. Peskin and N. Moiseyev, J. Chem. Phys. 99, 4590 (1993).

[27] K. Yajima, J. Math. Soc. Jpn. 29, 729 (1997).

[28] J. S. Howland, Indiana J. Math. 28, 471 (1979).

[29] G. Jolicard and J. P. Killingbeck, J. Phys. A 36, R411 (2003).

[30] R. Ketzmerick and W. Wustmann, Phys. Rev. E 80, 021117 (2009); 82, 021114 (2010).

[31] J. Ortigoso, M. Rodríguez, J. Santos, A. Karpati, and V. Szalay, J. Chem. Phys. 132, 074105 (2010).

[32] R. H. Young and W. J. Deal, J. Math. Phys. 11, 3298 (1970). 